\title{
Tratamento de fratura mandibular por projétil de arma de fogo com uso de fixador externo: relato de caso
}

\author{
Treatment for fracture mandibular of firearm with external fixation: case report
}

\author{
Igor Lerner Hora Ribeiro; Lucas Souza Cerqueira²; Fátima Karoline Almeida Dultra³; Joaquim Almeida \\ Dultra*; Bráulio Carneiro Júnior ; ; Roberto Almeida de Azevedos \\ ${ }^{1}$ Residente do Serviço de Cirurgia e Traumatologia Buco-Maxilo-Facial, UFBA/Hospital Santo Antônio/Obras \\ Sociais Irmã Dulce); Salvador, BA. ${ }^{2}$ Cirurgião-Dentista pela UEFS- Feira de Santana-BA, ex-estagiário em \\ Traumatologia Buco-Maxilo-Facial do HGE-BA. ${ }^{3}$ Doutoranda em Processos Interativos de Órgãos e Sistemas \\ PIOS/ICS; Mestre em Odontologia; Especialista em Cirurgia e Traumatologia Buco-Maxilo-Facial pelo Hospital \\ Santo Antônio(Obras Sociais Irmã Dulce)/UFBA. ${ }^{4}$ Mestre em Odontologia; Professor de Odontologia da UESB; \\ Preceptor do serviço de Cirurgia e Traumatologia Buco-Maxilo-Facial do Hospital Santo Antônio/Obras Sociais \\ Irmã Dulce)/UFBA. ${ }^{5}$ Mestre e Doutor em Odontologia; Professor adjunto da UFBA; Coordenador do serviço de \\ Cirurgia e Traumatologia Buco-Maxilo-Facial do Hospital Santo Antônio(Obras Sociais Irmã Dulce)/UFBA.
}

\begin{abstract}
Resumo
A violência e o uso indiscrimidado das armas de fogo, independente da classe social, tornaram os ferimentos por projétil de arma de fogo (PAF), comum nos grandes centros e geralmente acometem a região maxilofacial, principalmente o terço inferior da face. Nos ferimentos por PAF em face, predominam as fraturas cominutivas em mandíbula e a presença de focos infecciosos. Apesar das diversas possibilidades para tratamento das fraturas mandibulares, o uso de fixadores externos tornou-se uma opção válida principalmente nos casos de fraturas complexas associadas à um quadro infeccioso, mesmo sendo adaptados para o uso em face. O objetivo deste trabalho é apresentar um caso clínico de um paciente vítima de ferimento por PAF em face com fratura cominutiva em região sinfisária de mandíbula, admitido no serviço de Cirurgia Bucomaxilofacial do Hospital Santo Antonio - Salvador-BA. O tratamento proposto foi a redução incruenta da fratura e estabilização usando o sistema de fixação externa.

Palavras - Chave: Fratura da Base do Crânio.Traumatismos Mandibulares.Técnicas de Fechamento de Ferimentos.

Abstract

The violence and the indiscriminate use of firearms as, regardless of social class, injuries made by a firearm projectile common in large cities and usually involve the maxillofacial region, mainly lower part of the face. In the wounds for firearms in face, the communited fractures in jaw and the presence of infections focus predominat. Although the diverse possibilities for treatment of the mandibular fractures as the rigid internal fixation, the external fixation use mainly became a valid option in the cases of complex fractures associates with infection, exactly being adapted for the use in face. The aim of this work is to present case report of a patient victim of firearm in face with communited fractures in jaw, admitted to the Department of Oral and Maxillofacial Surgery of the Hospital Santo Antonio - Salvador-BA. It was opted as treatment the closed reduction of the fractures and stabilization using the system of external fixation.

Keywords: Skull Fracture, Basilar.Mandibular Injuries.Wound Closure Techniques.
\end{abstract}

\section{INTRODUÇÃO}

Os ferimentos por projétil de arma de fogo têm se tornado um problema de saúde pública mundial. 0 uso de armas de fogo afetam todos os indivíduos da sociedade apresentado índices estatísticos cada vez maiores ${ }^{1}$. Em $61 \%$ das vítimas, os ferimentos ocorreram na cabeça e/ou face, com maior incidência na região de mandíbula ${ }^{2}$, sendo a região de corpo mandibular a mais atingida ${ }^{1}$. As fraturas mandibulares geralmente são cominutivas, com pequenas e/ou múltiplas linhas de fratura, resultando em fragmentos ósseos na área atingida pelo agente traumático ${ }^{2}$. Trata-se de ferimentos

Recebido em 22/02/2012; revisado em 03/04/2012.

Correspondência / Correspondence: Igor Lerner Hora Ribeiro. Praça 12 de Abril, no21, Centro, CEP: 57.200.000, Penedo - Alagoas. E-mail: lernerigor@hotmail.com contaminados, cujo trajeto de penetração do projétil é extremamente imprevisível.

O uso de fixadores externos é mais uma opção para tratamento dos ferimentos por paf em mandíbula 3 , sendo uma escolha incomum no tratamento dessas fraturas em virtude do uso de placas e parafusos, além de ter indicação restrita. ${ }^{4}$. A utilização regular de fixadores externos para o tratamento da fratura na mandíbula começou durante a segunda guerra mundial ${ }^{4}$. Os fixadores tradicionais apresentam desvantagens significativas. Sistemas compostos por uma barra acrílica com pinos metálicos requerem materiais e equipamentos extras, os quais, uma vez instalados, não podem mais ser ajustados. O uso de fixadores projetados para tratar fraturas de punho embora rápido e de aplicação simples, são volumosos para usar e as 
formas dos pinos e das barras não são apropriadas para a mandíbula ${ }^{3}$.

Ao contrário do fixador externo tradicional, existem fixadores que são próprios para mandíbula, adaptando -se aos contornos desse osso, sendo mais leves, posicionados a apenas $1,0 \mathrm{~cm}$ da superfície da pele e sendo ajustáveis ${ }^{3}$. Como a barra é mais perto do osso do que em outros sistemas, promove mais estabilidade nos locais da fratura. Os pinos especiais de titânio possuem o comprimento correto da cabeça do parafuso para as regiões de parasínfise, corpo e do ramo da mandíbula. A combinação nova das braçadeiras para conectar a barra do fixador, pinos e todas as hastes de conexão adicionais de titânio é de um projeto recente ${ }^{3}$.

O pino de fixação externa pode ser usado nas fraturas que possuam sítios edêntulos ou com dentição inadequada, onde existem perdas ósseas secundarias às lesões por armas de fogo, fraturas cominutivas, infecções, fraturas patológicas ou osteomielite. O uso também é indicado em condições especiais como comprometimento das vias aéreas superiores, problemas nutricionais. Pode também ser utilizado nas fraturas das mandíbulas edêntulas atróficas ou nas fraturas mandibulares associadas com as do terço médio da face, quando um método rápido e simples para fixação é necessária ${ }^{5}$.

O objetivo deste trabalho é apresentar um caso clínico de um paciente vítima de ferimento por PAF em face com fratura cominutiva em região de sínfise mandibular, admitido no serviço de Cirurgia Bucomaxilofacial do Hospital Santo Antonio - SalvadorBA. Foi optado como forma tratamento a redução incruenta da fratura e estabilização usando o sistema de fixação externa.

\section{RELATO DE CASO}

Paciente feoderma, 54 anos, gênero masculino, sem patologias de base, foi atendido pelo Serviço de Cirurgia e Traumatologia Bucomaxilofacial do Hospital Santo Antônio-Salvador-BA, com história de ferimento por projétil de arma de fogo (PAF) em face após tentativa de suicídio com espingarda de socar pela boca. Apresentava dificuldade para alimentar-se e drenagem de secreção purulenta pelo orifício de entrada do PAF na região submentoniana, com evolução de 10 dias.

Ao exame físico (figuras 1 e 2) observou-se aumento de volume em região de mento, hiperemia local e lesão transfixante suturada em região submentoniana e assoalho bucal com sinais de infecção e deiscência, apresentava drenagem espontânea de secreções salivar e purulenta, mobilidade mandibular atípica à manipulação, relação intermaxilar alterada, edentulismo parcial, abertura bucal satisfatória e má higiene oral. Ao exame de tomografia computadorizada (figura 3) verificou-se fratura mandibular cominutiva em região sinfisária sem grande deslocamento e estilhaços de PAF associado.

Foi iniciado antibioticoterapia venosa seguido de procedimento cirúrgico procedimento cirúrgico para redução fechada e estabilização da fratura mandibular, sob anestesia geral, onde foram realizados irrigação abundante, desbridamento conservador da ferida com remoção de fragmentos do projétil e tecidos inviáveis, exploração cirúrgica das feridas intra e extra-oral e sutura. Avaliando as condições locais (infecção, edentulismo, cominuição, impossibilidade de bloqueio maxilo-mandibular (BMM) adequado, perda substancial de tecidos moles), história do trauma e evolução da doença, optou-se por redução incruenta da fratura e estabilização usando o sistema de fixação externa, através da adaptação de um fixador externo de punho. Foram então instalados oito pinos bicorticais, através de trocarter, sendo dois pinos em região pós-forame mentual bilateralmente e quatro em região de sínfise, a seguir, foi feito a união dos quatro pinos de cada lado através de braçadeiras e barra metálica.

No primeiro dia pós-operatório (DPO) notou-se ainda drenagem salivar através da pele por pressão da lingua em assoalho bucal, a comunicação foi tamponada com gaze vaselinada. No sétimo DPO foi observado fixador externo em posição (figura 4), discreta mobilidade mandibular à manipulação, ferimento intra-oral cursando com cicatrização por segunda intenção e sem sinais de infecção. Foi então realizado a união dos pinos e barra metálica de cada lado com resina acrílica (figura 5), visando maior estabilização da fratura, e irrigação de ferimento intraoral com soro fisiológico. Ao exame de imagem pósoperatório (figura 6) constatou-se o bom posicionamento dos pinos ósseos, alinhamento e contorno ósseo satisfatórios.

Três meses após a cirurgia, notou-se reparo mucoso e ósseo, sem sinais de infecção e sem sinais de mobilidade em sínfise mandibular, através de exame clínico-radiográfico, foi removido o fixador externo sob anestesia local. Um ano após a cirurgia o paciente retornou com queixas álgicas associada ao elemento 35 , o qual foi removido, cessando a dor do paciente. 0 mesmo encontra-se em proservação através de revisões semestrais, sendo a última realizada no vigésimo mês após a cirurgia, onde foi observado contorno mandibular mantido, ausência de mobilidade atípica e funções da mandíbula preservadas.

\section{DISCUSSÃO}

As fraturas cominutivas da mandíbula têm sido tratadas de diversas formas, incluindo redução aberta com placas e parafusos, uso de fixação interna com fios de aço, redução fechada com utilização de BMM e, em alguns casos, através de redução incruenta com uso de fixadores externos ${ }^{6}$. 


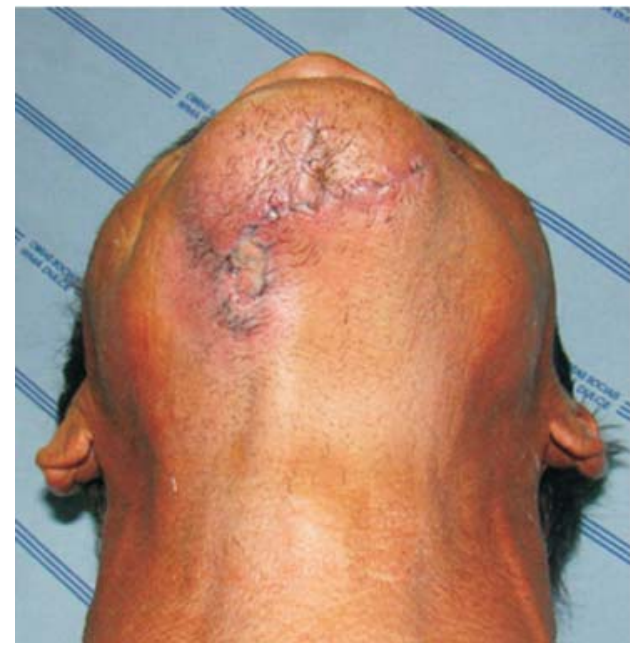

Figura 1 - Exame físico extra-oral.

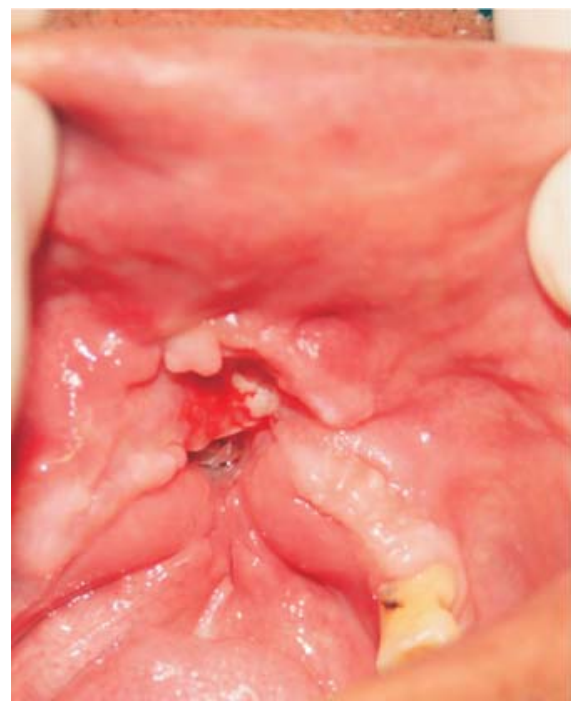

Figura 2 - Exame físico intra-oral.

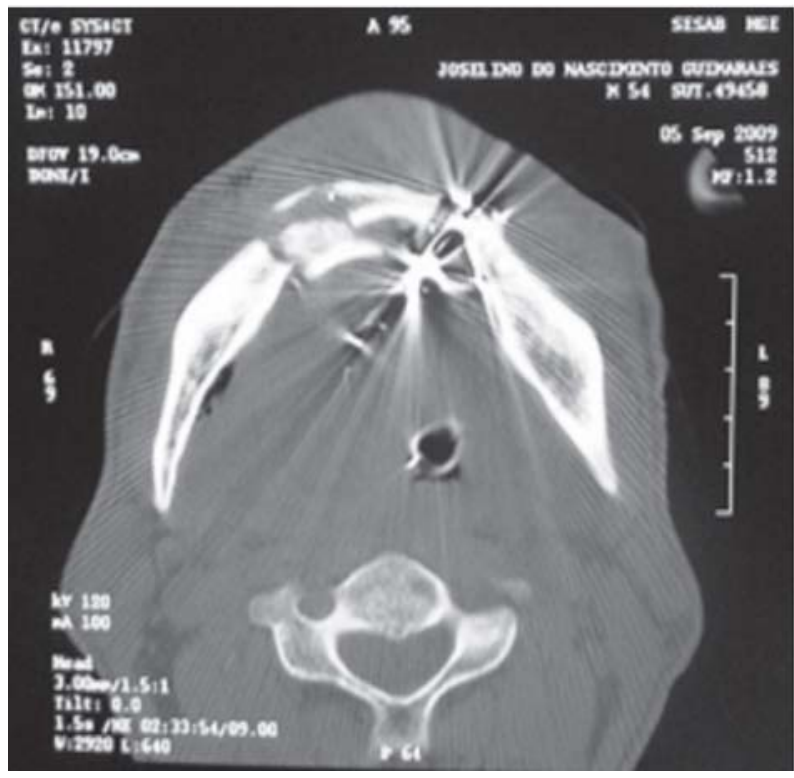

Figura 3 - Tomografia computadorizada pré-operatória.

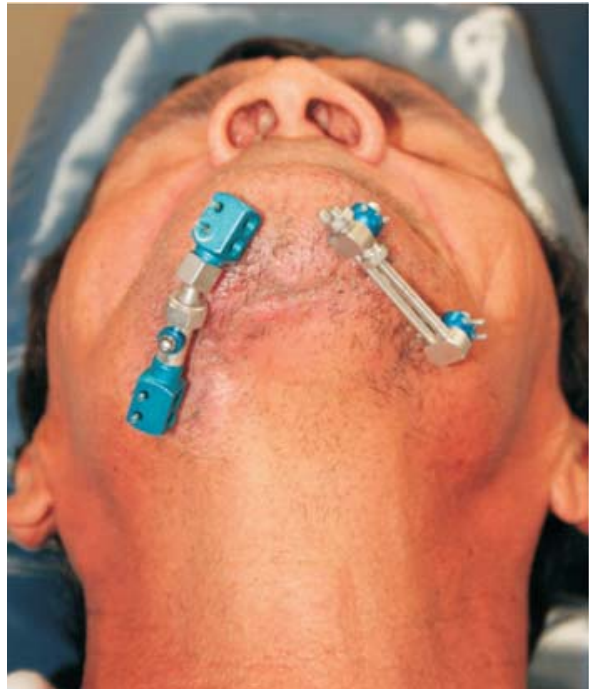

Figura 4 - Sétimo dia pós-operatório.

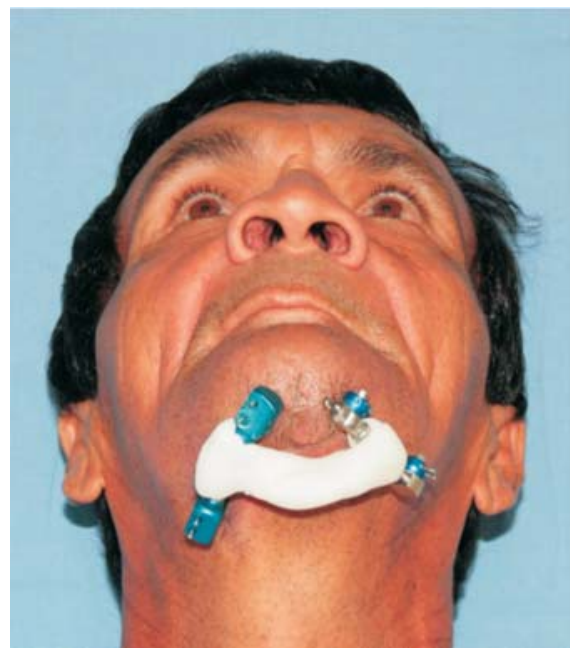

Figura 5 - União de fixadores externos com resina acrílica.

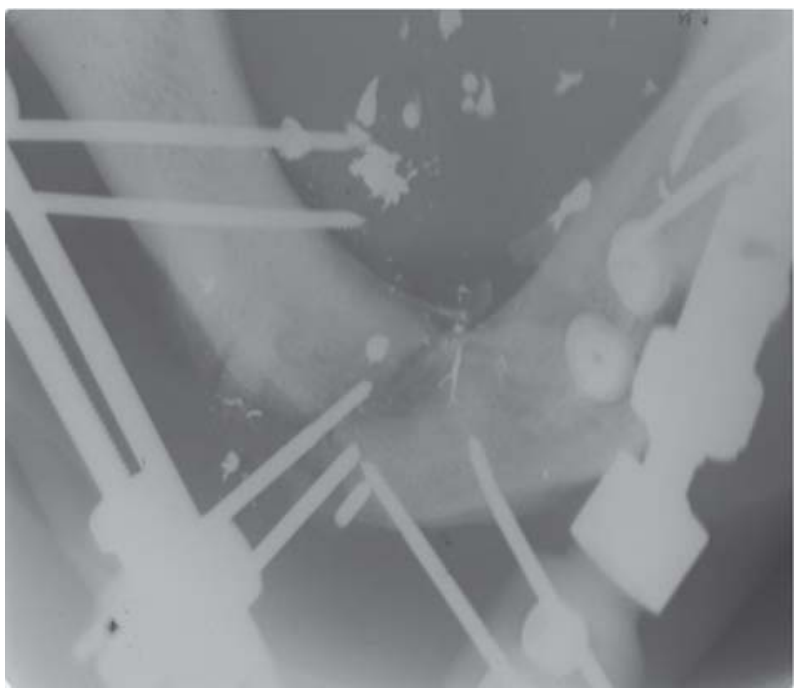

Figura 6 - Radiografia oclusal pós-operatória. 
Os fixadores externos provaram ser um método popular de tratar os ferimentos por arma de fogo. Sistemas incorporando uma barra acrílica com metal e pinos exigem materiais e equipamentos extras ${ }^{3}$, tais sistemas feitos de titânio são biocompatíveis e garante resistência e rigidez ${ }^{7}$. A fixação externa de fraturas da mandíbula é uma técnica em que os segmentos são manipulados no lugar pelos pinos e fixados com conectores ${ }^{4}$. Frequentemente considera-se um subtipo de redução fechada mesmo quando uma técnica aberta é usada para colocar o fixador externo, nenhum corpo estranho é introduzido no local da fratura ${ }^{3}$. Fornece uma fixação semi-rígida aos segmentos mandibulares fraturados ${ }^{4}$, mesmo assim, tal fixação garante a estabilidade adequada dos fragmentos ${ }^{7}$.

Apesar de poucos casos avaliados com sistema de fixação externa em mandíbula humana, os fixadores externos parecem favoráveis para a osteosíntese ${ }^{4}$. Geralmente as indicações para a fixação externa é quando o bloqueio maxilomandibular não é adequado por causa de ausências dentárias, fraturas cominutivas, infecção presente, tecido mole de recobrimento insuficiente ou contra indicação devido razões médicas concomitantes ${ }^{8}$, além de grandes perdas ósseas causadas por ressecções de tumores, infecções levando a grandes sequestros ósseos e traumas graves com perda de substância ${ }^{6}$.

O uso dos fixadores externos apresenta a vantagem de não ser necessário descolamento periosteal e o potencial prejuízo na vascularização de um osso já comprometido. Além disso, as incisões são menores, e a sua instalação pode ser feita em poucos minutos, algumas vezes, sob anestesia local ${ }^{6}$. O fixador externo tem muitas vantagens devido sua versatilidade e simplicidade de uso. Quando possível, pode se feito sob anestesia local nos casos onde a anestesia geral for contra-indicada ou não disponível. Outra vantagem está no controle dos fragmentos ósseos ao manipular os pinos e os conectores após a avaliação radiográfica com ou sem o uso da anestesia local. Com esse sistema, a boca do paciente pode ser aberta durante o tratamento da fratura, a higiene oral e a nutrição do paciente são melhoradas e o trismo devido à fibrose e a cicatrização das feridas, são reduzidos ${ }^{3}$. Infelizmente, devido a posição, os dispositivos de fixação externa são frequentemente incômodos para pacientes, por ser inestético do ponto de vista social ${ }^{8}$.

$\mathrm{Na}$ literatura, a taxa de complicações relacionadas ao tratamento de fraturas cominutivas de mandíbula chega a $13 \%$. As variáveis citadas mais relacionadas com o desenvolvimento das complicações são a técnica cirúrgica utilizada, a etiologia do trauma e o tempo até a instituição do tratamento. Dentre estas variáveis, merecem menção as taxas de complicação, comparativamente maiores, relacionadas ao uso dos sistemas de fixação externa $(35,2 \%)$ e os traumas causados por armas de fogo (27,8\%). Dados demonstram que as lesões mais graves (causadas por armas de fogo) geralmente levam a maiores dificuldades de tratamento, necessitando opções menos efetivas (fixadores externos), mas que geralmente são as únicas alternativas possíveis para tais casos ${ }^{6}$.

No estudo de CORNELIUS et al., (2009) ${ }^{4}$, observou-se uma taxa de complicação elevada de até $35 \%$ com o uso de fixador externo. E no trabalho de ELLIS III et al., (2003) ${ }^{9}$, houve uma taxa de complicação superior como uso de fixadores externos em relação aos pacientes tratados com redução aberta e fixadores internos. Entretanto, em ambos os estudos, os pacientes tratados com os fixadores externos tiveram os ferimentos mais severos, como feridas oriundas de arma de fogo e colisões de veículo motorizado.

Das infecções pós operatórias, a celulite em torno dos pinos, as não uniões, as más oclusões, e o afrouxamento do pino são problemas frequentes com esta técnica de fixação. Outras complicações incluem lesão ao nervo alveolar inferior, especialmente em mandíbulas atróficas. Além de dano à glândula parótida e formação de mucoceles. A queimadura da pele pela polimerização acrílica pode ocorrer se as precauções e a técnica apropriadas não são seguidas ${ }^{4}$. Neste trabalho não foram observadas complicações pós operatórias, foi constatado a união dos fragmentos e a ausência de focos de infecção. Nenhum problema relacionado com o uso do equipamento foi verificado.

Mais recentemente, redução aberta usando placas e/ou parafusos foi indicada para fraturas cominutivas, mesmo contrariando conceitos básicos da cirurgia maxilofacial onde os estados das fraturas cominutivas devem ser tratados por redução fechada para impedir a escassez do suprimento sanguíneo. Entretanto, a literatura sugere que o impedimento de alguma fonte de vascularização não conduz à incidência aumentada de infecção, contanto que a estabilização dos fragmentos ósseos seja conseguida ${ }^{9}$.

O BMM com parafusos de fixação intermaxilar (FIM) é um excelente método para tratar fraturas mandibulares provocadas por ferimentos balísticos simples. Os sistemas externos são mais uma alternativa no tratamento de fraturas mandibulares mais complexas ${ }^{3}$. Segundo GIBBONS et al., (2011) ${ }^{3}$, a colocação de um fixador externo e de quatro parafusos de FIM é uma técnica mais rápida. A FIM pode ser usada para estabilizar e reduzir temporariamente fraturas mandibulares durante a colocação do fixador externo. Durante o tratamento podem ser usados elásticos para guiar a oclusão correta enquanto o fixador externo mantem as áreas fraturadas em sua posição correta.

Atualmente, o tratamento considerado padrão para as fraturas complexas de mandíbula é o uso de fixação interna rígida e uso de enxertos ósseos. Porém, o uso de fixadores externos como opção de tratamento para tais lesões mandibulares ainda tem seu espaço, particularmente em lesões associadas a quadros 
infecciosos, com presença de extensa reação inflamatória e áreas de sequestro ósseo, assim como em lesões provocadas por armas de fogo, com extensa destruição óssea e de partes moles. A fixação externa com fixadores ortopédicos deve ser lembrada como opção válida e mais acessível para o tratamento temporário de lesões complexas, já que o dispositivo utilizado está disponível no sistema público de saúde, não necessitando, portanto, de material específico ${ }^{6}$.

\section{CONSIDERAÇÕES FINAIS}

Nas fraturas cominutivas mandibulares oriundas de ferimentos por PAF não existe consenso para uma única forma de tratamento. A utilização dos fixadores externos é uma opção de tratamento viável e efetiva quando bem indicada, como pôde ser observado no caso clínico descrito, onde foi conseguido satisfatório contorno mandibular, ausência de mobilidade atípica, boa relação intermaxilar e funções mandíbulares preservadas. Conclui-se que a estabilização de fraturas cominutivas por PAF pelo método de fixação externa é eficiente quando respeitadas as técnicas de uso e os protocolos de antibioticoterapia

\section{REFERÊNCIAS}

1- SILVA, J.J.,et.al. Lesão por arma de fogo em terço inferior de face de crianças: relato de caso. Rev.Cir.Traumatol. BucoMaxilo-Facial., Camaragibe, v.4, n.3, p. 163-168, 2004.

2- PEREIRA, C.C.S. et.al. Fratura mandibular por projétil de arma de fogo. Rev. Cir. Traumatol. Buco-Maxilo-Facial., Camaragibe. v.6, n. 3, p. $39-46,2006$.

3- GIBBONS, A.J.; MACKENZIE, N.; BREEDERVELD, R.S. Use of a custom designed external fixator system to treat ballistic injuries to the mandible. Int. J. Oral Maxillofac. Surg., Copenhagen, v.40, n. 1, p. 103-105, 2011.

4- CORNELIUS, C.P.; AUGUSTIN, J.B.; SAILER, L.K. External pin fixation for stabilization of the mandible-comeback of a method: historical review and first experiences with the 'mandible external fixator'. Int. J. Oral Maxillofac. Surg., Copenhagen, v.13, n.1, p. 1-14, 2009.

5- MILORO, M. et.al. Princípios de Cirurgia Bucomaxilofacial. 2 ed. São Paulo: editora Santos, 2008.

6- TENIUS, F.P. et.al. Uso de fixadores ortopédicos para fixação externa em fraturas complexas de mandíbula: estudo retrospectivo. Rev. Soc. Bras. Cir. Craniomaxilofac.,São Paulo, v.9, n. 2, p. 25-30, 2006.

7- CASCONE, P. et.al. Rigid versus semirigid fixation for condylar fracture: Experience with the external fixation system. Int. J. Oral Maxillofac. Surg., Copenhagen, v.66, n.2, p. 265-271, 2008.

8- BRAIDY, H.F.; ZICCARDI, V.B. External fixation for mandible fractures. Atlas oral Maxillofac. Surg. Clin. North. Am. Philadelphia PA, v.17,n.1, p. 45-53, 2009.

9- ELLIS, E 3rd; MUNIZ, O.; ANAND; K. Treatment considerations for comminuted mandibular fractures. Int. J. Oral Maxillofac. Surg., Copenhagen, v.61, n.8, p.861-870, 2003. 